\title{
Actuation Design Methodology for Haptic Interfaces and Rehabilitation Systems
}

\author{
Michele Folgheraiter \\ Robotics and Mechatronics Department \\ Nazarbayev University, School of Science and Technology \\ 53 Kabanbay Batyr Ave, Astana, Kazakhstan \\ michele.folgheraiter@nu.edu.kz
}

\begin{abstract}
This paper introduces a methodology and a software framework intended to optimize and speed up the design process of a haptic interface or a rehabilitation system. Starting from an initial mechanical design the procedure allows to export the kinematic and dynamic properties of the robotic system in a simulation environment. The software receives as additional input the Cartesian or joints trajectories and generates as output the required torques at the joints. From the recorded measurements the program extracts the torque ranges necessary to choose a suitable actuation system for the robot. The possibility to run the simulation in batch modality allows also to define different optimization techniques that may be used to reduce the overall system weight or increase its payload.
\end{abstract}

Keywords-Actuation System Design, Actuation System Optimization, Haptic interface, Rehabilitation System.

\section{INTRODUCTION}

Designing the actuation system for a robot is a crucial step during the machine development. This phase is even more critical if the robotic system is represented by a haptic interface or a rehabilitation system that are meant to operate in strict contact with the human body. Generally, the design process takes into account a realistic model of the limb interacting with the interface [1], [2] and includes as set of simulations intended to study the coupled interface-limb system. The result of these simulations allow to fix important requirements necessary to design the interface. Among them the number of Degrees of Freedom (DOF), the joints type and configuration, and the links dimensions. When the kinematic of the interface is ready a proper dynamic model [3] needs to be formalized and computed. This is demanded to find out other important design elements like the optimal actuators displacement and dimensioning. The principal goal of the designer at this point is to obtain an interface having good performances in term of displacement accuracy, sufficient force feedback and resolution, lower energy consumption, system's lightweight, backdrivability, etc.. This, in general, is not a trivial task due to the fact that, in most of the cases, an improvement in one direction brings inevitably a worsening in the others. Finally when the interface is implemented it is necessary to conduct a long test phase where the control system is finely tuned and the comfort and effectiveness of the prototype is measured by mean of a suitable metric [4], [5].

In the case that the kinematic structure of the robot is relative simple it is possible to represent the optimization problem with a pure analytical approach and use a global or local optimum design process [6]. However, when the complexity of the system increases or when obtaining a pure analytical model results tedious, alternative methods need to be found. The scope of this work is to develop a methodology that will support choosing and dimensioning the actuation system of a human-machine interface having a complex and redundant kinematics. The method takes into account: the interface parametric model exported from the CAD program, a performances metric, and an optimization procedure. The optimization process does not starts from scratch, due to the huge dimension of the search-space (e.g all the possible kinematics configurations, the actuators locations, the links dimensions, the weights distribution, etc.), but it starts from a "good initial point". The initial design is normally developed by an experienced mechanical designer. Thanks to its knowledge an expert is able to apply a first important screening of the all technically feasible solutions and configurations. It is also possible to reduce even further the research space by defining a restricted set of changeable parameters. One may choose for the final design e.g. only the most critical parameters. Furthermore, in order to make the optimization procedure possible, it is required to define different performances metrics. Between the possible options we can chose: the energy consumption, the workspace dimension, or the force isotropy.

The rest of the paper is organized as following: next section describes the method we propose and does some considerations about how to characterize and compare different actuators, section III introduces the simulation framework, section IV brings some preliminary results, and finally section $\mathrm{V}$ draws the conclusions and indicates possible future developments.

\section{THE METHODOLOGY}

The method we developed is mainly intended to optimize the actuation system of a haptic interface. However, it can also be adapted to minimize its energy consumption, to limit the movement restriction when interacting with the human limb, and to optimize its kinematic architecture. The optimization operates in discrete domain and starts from an initial hardware configuration $D_{i}$. After a trajectory is the defined in the interface's Cartesian workspace $[P(t), \dot{P}(t), \ddot{P}(t)]$ (see Fig. 1) or in the joint space $\left[q_{i}(t), \dot{q}_{i}(t), \ddot{q}_{i}(t)\right]$, the software computes the required torque $\tau_{i}(t)$ for each joint, and finally evaluates the chosen cost function $E_{i}$ (in our specific case the energy consumed to actuate the reference trajectory). This sequence of steps is repeated for each hardware configuration and terminates only when all the configurations are explored and the best solution is chosen. 
It is worth to mention here that when the optimization process is finished, the evaluation of an expert designer is still required to verify that the solution is realizable.

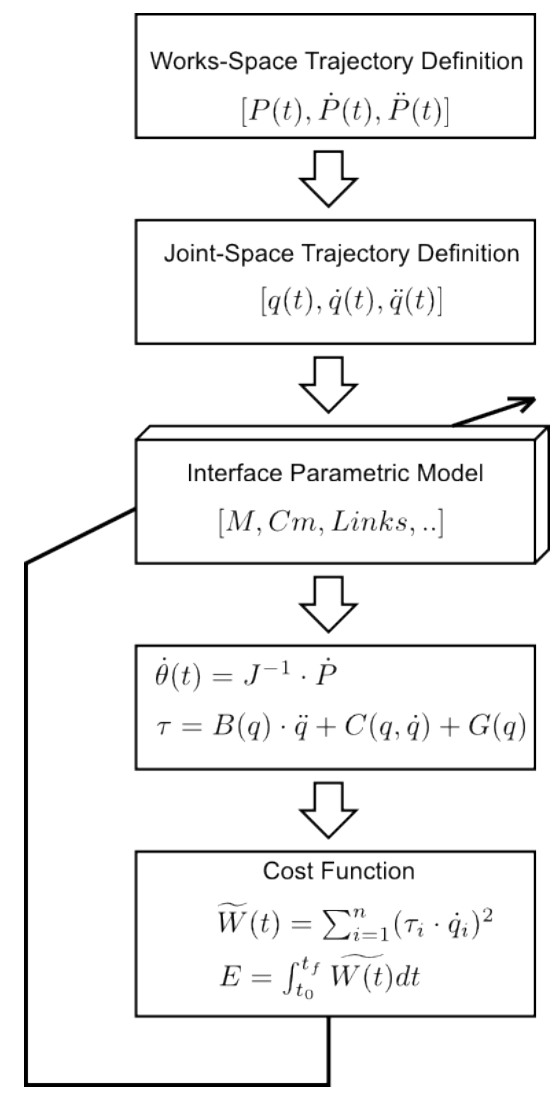

Fig. 1. Optimization scheme based on the interface parametric model.

\section{A. Search Space}

The discrete space where to search the optimal solution is the set $\mathbf{D}=\left\{D_{1}, \ldots, D_{m-1}, D_{m}\right\}$ of all the possible hardware configurations. The single configuration $D_{i}$ in its turn consists of an open kinematics chain $K_{i} \in \mathbf{K}=\left\{k_{1}, \ldots, k_{m-1}, k_{m}\right\}$ in which each $j^{t h}$ DOF has a defined actuator associated $a_{j}$ that can be chosen from a set of $p$ possible technical variations $\mathbf{A}=\left\{a_{1}, \ldots, a_{p-1}, a_{p}\right\}$. Therefore an hardware configuration $D_{i}$ can be represented by a pair $(K, A K)_{i}$ where $K$ represents a specific kinematics chain and $A K$ is defined by a permutation of $k$ actuation elements $\left\langle a_{1}, \ldots, a_{k-1}, a_{k}>\right.$ where $a_{i} \in \mathbf{A}$, repetitions are allowed, and the order of the element sequence is also significant. In general defined the kinematic chain $K$ and the set $\mathbf{A}$, and assuming $k \leq p$, we have a total of $\frac{p !}{(p-k) !}$ possible configurations for the actuation system.

Fig. 2 depicts a possible configuration for the hardware, where $L_{i}$ represents the $i^{t h}$ link that is rigidly connected with the joint $J_{i}$ which in its turn is governed by the actuator $a_{i}$. The actuator $a_{i}$ can be described by a set of parameters: the electrical power $W_{i}$, the mass $M_{i}$, and the rotor inertia $I_{i}$. Defined a certain pose $\bar{q}$ for the kinematics chain, and

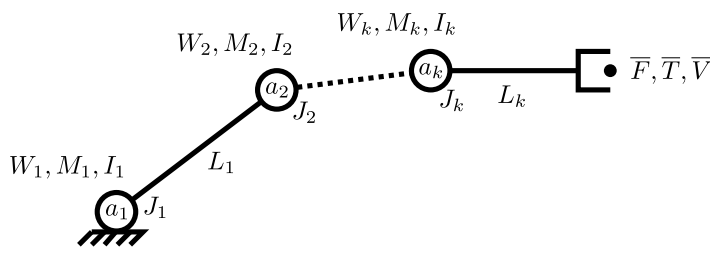

Fig. 2. An example of hardware solution, each joint of the kinematic chain is associated with an actuator having a defined electrical power $W$, mass $M$ and output inertia $I$

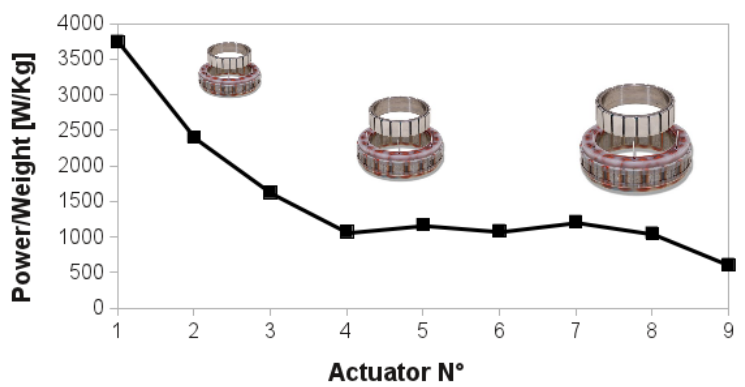

Fig. 3. Motors' performance, in term of power to weight ratio, relative to nine motors of different dimensions produced by the Robodrive $(\mathrm{GmbH})$ company

assuming to evaluate the system in a certain instant $t_{s}$, it is possible to calculate the force $\bar{F}$ and torque $\bar{T}$ generated at the end-effector, and relative to the Cartesian space, as $\left(\bar{F}\left(t_{s}\right), \bar{T}\left(t_{s}\right)\right)=\mathbf{J}\left(\bar{q}\left(t_{s}\right)\right) \cdot \bar{\tau}$, where $\mathbf{J}$ is the geometric Jacobian matrix calculated at the specific pose $\bar{q}\left(t_{s}\right)$. Furthermore, assuming that the velocity of the end-effector is represented by the vector $\bar{V}=(\dot{\bar{X}}, \dot{\bar{\theta}})$ it is possible to calculate the mechanical power at the instant $t_{s}$ as the scalar product $\left.W_{M}\left(t_{s}\right)=\bar{V}\left(t_{s}\right) \cdot \overline{(} \bar{F}\left(t_{s}\right), \bar{T}\left(t_{s}\right)\right)^{T}$

\section{B. Actuators Set and its Characterization}

As a prerequisite to start the optimization procedure it is necessary to define the set of the possible kinematic chains $\mathbf{K}$ and the set of the possible actuators A. Furthermore, due to the fact that it is not realist to consider any possible realizable kinematics chain and any commercially available actuator, it is also necessary to limit these two sets in dimension with the support of an expert.

To select a set of possible actuators one has at first to define some performances measurement that facilitates the comparison of different configurations and technologies. In the case of an electrical motor a good metric is represented by the power to weight ratio $P T W=\frac{W_{i}}{M_{i}}=\left[\frac{W}{k g}\right]$. As an example Fig. 3 reports the power-to-weight to ratio of a set of brash-less motors commercialized by the company Robodrive $(\mathrm{GmbH})$. As it possible to notice the curve is not linear, but more similar to the function $y=\frac{1}{K \sqrt[n]{x}}$.

Furthermore, it is possible to notice that the bigger is the motor the smaller is the ratio between these two quantities. This shows that for this particular technology and size range 


\begin{tabular}{l|cc} 
Actuator & PTW $\left[\frac{W}{k g}\right]$ & PTWI $\left[\frac{W}{k^{2} m^{2}}\right]$ \\
\hline$a_{1}$ & 111 & $2.6 \cdot 10^{6}$ \\
$a_{2}$ & 496 & $0.27 \cdot 10^{6}$ \\
$a_{3}$ & 1453 & $2.9 \cdot 10^{6}$
\end{tabular}

TABLE I. COMPARISON, IN TERMS OF POWER-TO-WEIGHT RATIO AND POWER-TO-WEIGHT-INERTIA RATIO, BETWEEN THREE DIFFERENT ACTUATORS

\begin{tabular}{lllc}
\hline Quantity & Description & Ref. System & Units \\
\hline CS1_POS_i & $1^{\text {st }}$ Frame Position $i^{t h}$ link & World & $\mathrm{m}$ \\
CS1_ROT_i & $1^{\text {st }}$ Frame Orientation $i^{t h}$ link & World & degrees \\
CS2_POS_i & $2^{n d}$ Frame Position $i^{t h}$ link & World & $\mathrm{m}$ \\
CS2_ROT_i & $2^{n d}$ Frame Orientation $i^{t h}$ link & World & degrees \\
COG_POS_i & Center of Mass $i^{t h}$ link & World & $\mathrm{m}$ \\
INERTIA_COG_i & Inertia Tensor $i^{t h}$ link & World & $\mathrm{kg} * \mathrm{~m}^{2}$ \\
MASS_L_i & Mass $i^{t h}$ link & None & $\mathrm{kg}$ \\
i_CAD_STL & $i^{t h}$ link CAD Model & World & None \\
TABLE II. & LIST OF KINEMATIC AND DYNAMIC PROPERTIES RELATIVE \\
TO THE $i^{t h}$ LINK. QUANTITIES WERE EXPORTED IN MATLAB USING THE
\end{tabular}
CAD-2-SIM SOFTWARE FRAMEWORK

smaller motors have better performances than bigger one. Another important parameter that characterizes an actuator is the rotor inertia $I_{i}$, one can also think to include this parameter and consider the quantity $P T W I=\frac{W_{i}}{M_{i} \cdot I_{i}}=\left[\frac{W}{\mathrm{~kg}^{2} \mathrm{~m}^{2}}\right]$. Using this metrics we can for example compare $a_{1}$, a $6 \mathrm{~W}$ DC motor from Maxon $(\mathrm{GmbH})$ weighting $54 g$ and having a rotor inertia of $4.1 \mathrm{gcm}^{2}$ with $a_{2}$, a $70 \mathrm{~W}$ brush-less motor from the same company weighting $141 \mathrm{~g}$ and having a rotor inertia of $181 \mathrm{gcm}^{2}$ with $a_{3}$, a $125 \mathrm{~W}$ brush-less motor from Robodrive $(\mathrm{GmbH})$ weighting $86 \mathrm{~g}$ and having a rotor inertia of $49 \mathrm{gcm}^{2}$. Table I reports the comparison of this three actuators in terms of PTW and PTWI ratios. How it is possible to notice using this particular metric $a_{3}$ represents the optimal choice among the three.

\section{Simulation FramewORK}

To test our methodology we defined a simulation environment that allows to conduct different kind of experiments and that, due to its high reconfigurability, facilitates the implementation of different optimization algorithms. The model was implemented in Matlab-Simulink and the SimMechanics library. The open source CAD-2-SIM software [7] was used to export the kinematic and dynamic properties of the designed interface directly from the 3D-CAD model that was developed in SolidWorks. Table II reports these quantities together with a brief description. It is worth to notice that in representing the kinematic model we used a double frame convention, i.e. each link is described by two reference systems (CS1 and CS2) located at the beginning and at the end of the structure and with $z$-axis having direction coincident with the direction of the rotational or prismatic axis of the correspondent joint.

The initialization phase of the simulation is performed directly in the Matlab workspace, where all the necessary model's parameters are defined in advance. This allows to conduct a series of simulations (i.e. calls to the Simulink model), having different initial parameters and boundary conditions, in a complete batch modality. Using this approach, as

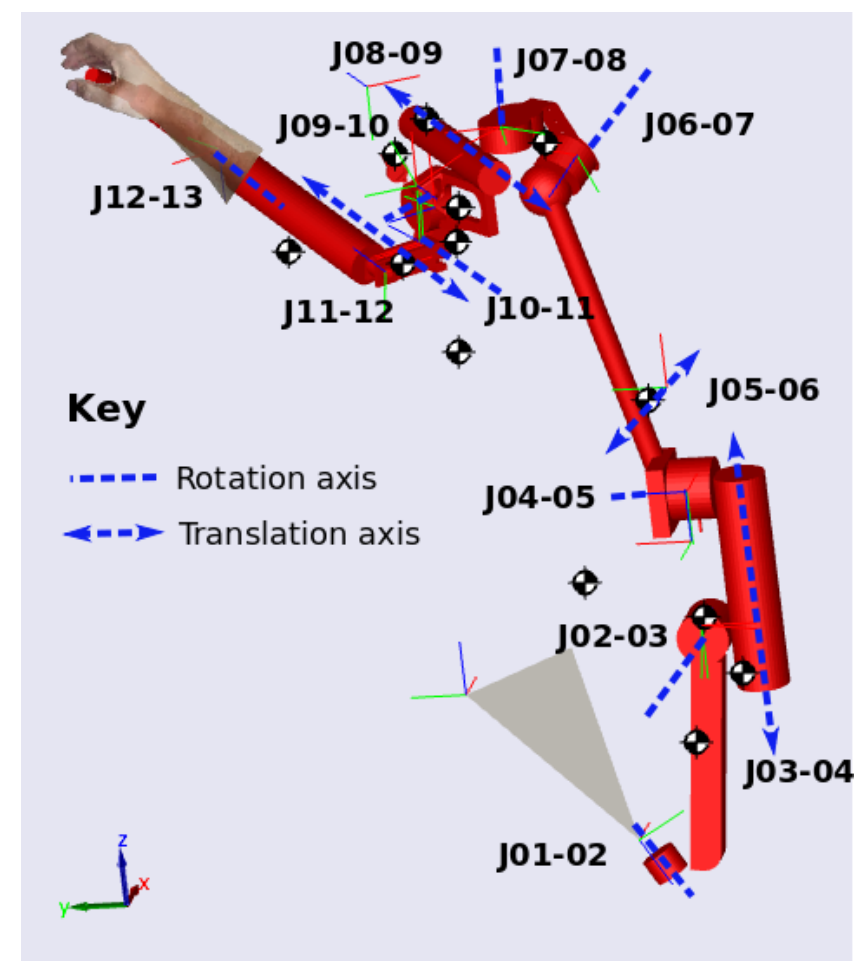

Fig. 4. SimMechanics model of a complex haptic interface having 12 DOF. The 3D representation of the mechanical parts and the dynamic properties were directly exported from the CAD using the CAD2SIM software. In blue color are indicated the rotational and prismatic joint's axes.

an example, it is possible to compute the interface workspace for different links dimension by different calls to the Simulink model. This can be used e.g. to optimize the workspace of the interface by proper dimensioning the mechanical parts. Figure 4 depicts the interface model represented using the SimMechanic visualization functionality. How it is possible to notice each link is furnished of three reference systems, two representing the starting and the ending points of the link, and a third one with origin in the center of mass of the link (small black spheres in Figure 4). Due to the fact that in the CAD design a link is normally constituted of different parts, for simplicity, we only represented the most significant one. This explains the fact that some of the centers of mass are located outside the robot's body. To notice also that the masses and the inertia matrices are all correctly included in the model even if not graphically represented.

Each joint represented in the SimMechanics has the possibility to be controlled in position or torque. In our case the second modality was chosen in order to have the possibility to measure the amount of torque (or force) required during the motion (or during a static pose). For this purpose a PID controller was integrated in each joint (see Fig. 5). This required an attentive tuning where all the PIDs' constants were adjusted in order to have good tracking position performances for each joint. All the variables acquired during the simulation (e.g. joint's torque, position, velocity, acceleration etc.) were automatically exported in the Matlab's workspace after the simulation was 


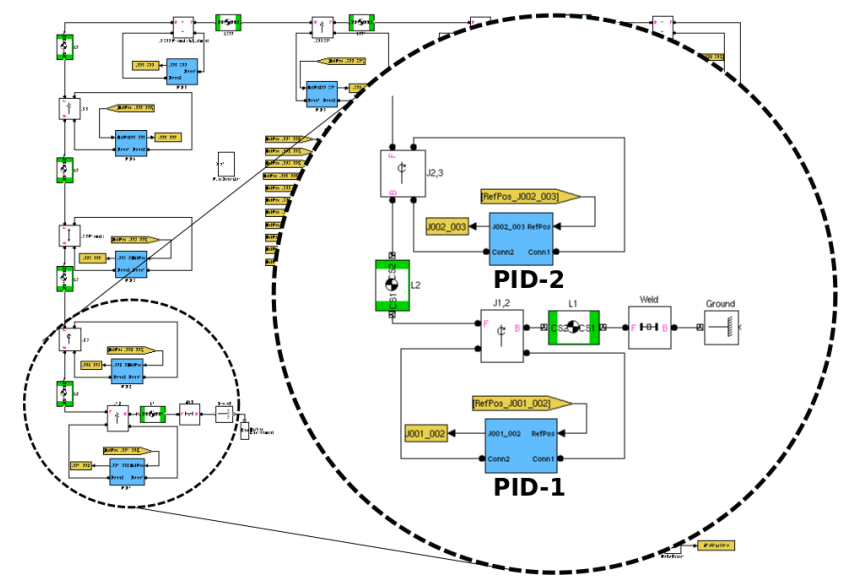

Fig. 5. Integrated with the Simulink model of the robot a set of PID controllers ensure that the position trajectories are correctly followed.

\begin{tabular}{llll}
\hline Link & Mass & Link & Mass \\
\hline L_01 & $3.59 \mathrm{Kg}$ & L_10 & $1.8 \mathrm{Kg}$ \\
L_02 & $1 \mathrm{Kg}$ & L_11 & $0.91 \mathrm{Kg}$ \\
L_03 & $0.94 \mathrm{Kg}$ & L_12 & $0.73 \mathrm{Kg}$ \\
L_04 & $1.2 \mathrm{Kg}$ & L_13 & $0.82 \mathrm{Kg}$ \\
L_05 & $1.2 \mathrm{Kg}$ & L_14 & $0.82 \mathrm{Kg}$ \\
L_06 & $0.4 \mathrm{Kg}$ & L_15 & $0.35 \mathrm{Kg}$ \\
L_07 & $0.15 \mathrm{Kg}$ & L_16 & $0.41 \mathrm{Kg}$ \\
L_08 & $0.2 \mathrm{Kg}$ & L_17 & $0.16 \mathrm{Kg}$ \\
L_09 & $3 \mathrm{Kg}$ & L_18 & $0.3 \mathrm{Kg}$ \\
\hline & & Total Weight & $17.98 \mathrm{Kg}$ \\
\hline
\end{tabular}

TABLE III. ESTIMATION OF THE LINKS' MASSES OF A POSSIBLE ACTUATED INTERFACE

terminated. This allowed us to elaborate all the data and plot the results via scripts that were based on the rich Matlab functions library.

\section{PREliminary Results}

A first series of simulations were intended to test the overall software framework, the joints' torque/force were measured during the execution of a quasi-static movement (slow speed and acceleration). Table III reports the values of the link's masses.

As a reference trajectories a set of sinusoids were defined with a period multiple of a basic time constant Tbasic $=$ $0.78 s$ (see Figure 6). More precisely starting from the last joint (wrist) we proceeded toward the first one (back) doubling each time the period of the reference sinusoidal trajectory. This was done in order to increase the coverage of the workspace, i.e. in this manner the same joint configuration will repeat only after a time interval of $800 \mathrm{~s}$. It is clear that the more we get near to the first joint of the kinematic chain the more the quasi static assumption is verified due to a slower trajectory.

The simulations results, in terms of computed joints' torques, are reported in Figure 7. How it is possible to notice the joints located in the back are the one that require higher torque to operate. This result make sense if we consider that they have to move most of the interface structure. However, the plots show also unintuitive results, e.g. the joint $J 002$ requires more torque in comparison with the joint J001_002

\begin{tabular}{lccc}
\hline Joint & Torque/Force Min & Torque/Force Max & Torque/Force \\
\hline J01_02 & -11.7 & 35.2 & $+/-35 \mathrm{Nm}$ \\
J02_03 & -11.2 & 62.4 & $+/-62 \mathrm{Nm}$ \\
J03_04 & 73.4 & 125 & $+125 \mathrm{~N}$ \\
J04_05 & -42 & 10 & $+/-42 \mathrm{Nm}$ \\
J05_06 & -50 & 47 & $+/-50 \mathrm{~N}$ \\
J06_07 & -26 & -0.9 & $+/-26 \mathrm{Nm}$ \\
J07_08 & -10 & 14 & $+/-14 \mathrm{Nm}$ \\
J08_09 & -17 & 24 & $+/-24 \mathrm{~N}$ \\
J09_10 & -3 & 1 & $+/-3 \mathrm{Nm}$ \\
J10_11 & -4 & 1.4 & $+/-4 \mathrm{Nm}$ \\
J11_12 & -4 & 28 & $+/-28 \mathrm{~N}$ \\
J12_13 & -1 & 0.95 & $+/-1 \mathrm{Nm}$ \\
\hline
\end{tabular}

TABLE IV. RANGES OF THE TORQUE/FORCE FOR EACH JOINT, CALCULATED IN QUASI STATIC CONDITIONS (SLOW JOINTS MOVEMENTS)

even if is more near to the end-effector. This depends on the fact that due to its orientation it is more subjected to the gravitational force. How it is possible to notice also the prismatic joint J003_004 needs a big force, however due to the fact that most of the time it has its axis parallel to the $z$-axis of the world frame, it is easy to think at a mechanism, e.g. based on springs, to compensate the static force. In this last case the actuator will be dimensioned only to generate the force to accomplish the dynamic motion (overcome the inertia effects of the structure). A complete range of the torques/forces required at each joint is resumed in table IV

\section{Conclusions}

This paper proposes a methodology based on a integrated CAD-simulation environment intended to help the designer to dimension the actuation system of a complex robotic system like a haptic interface or a rehabilitation system. Preliminary results demonstrated the main functionalities and capabilities of the software framework. In particular the kinematic and the dynamic model of the robot together with its visual representation was exported from the CAD design using the software CAD-2-SIM and loaded by automatized scripts in Matlab environment. The Simulink model integrates a set of joint controllers able to follow the target trajectories intended to cover as much as possible the interface workspace. The data relative to the actual joint position, velocity, acceleration, and torque/force are recorder and elaborated in order to obtain important information to dimension the actuation system of the interface. Future work will be dedicated to use the simulation environment in batch modality and perform a sequence of simulations intended to optimize different interface's parameters. As an example together with the dimensioning of the actuation system will be interesting to find out the optimal displacement for the actuators along the kinematic chain. This of course requires to carefully fix the set of possible allowed positions in order to limit the amount of computation needed to conduct the simulations.

\section{REFERENCES}

[1] N. Klopcar and J. Lenarcic, "Kinematic model for determination of human arm reachable workspace," Meccanica, no. 40, pp. 203-219, 2005. 

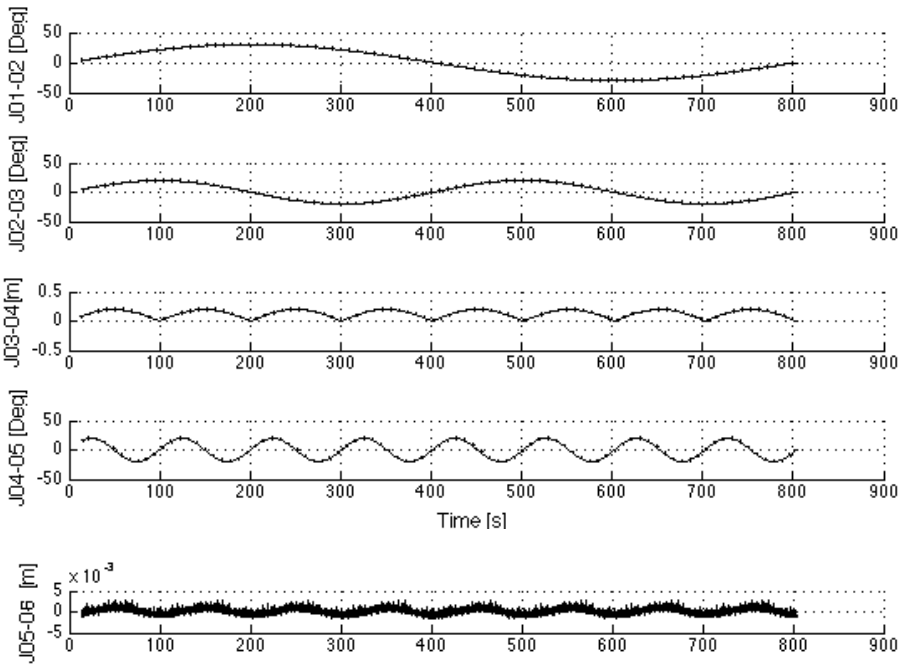

물.

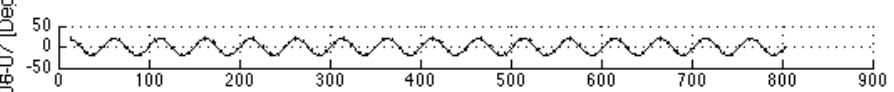

总

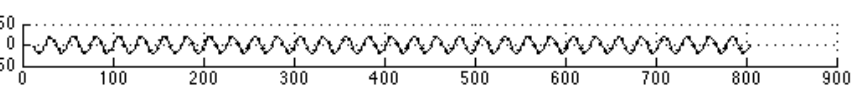

昰 0.5
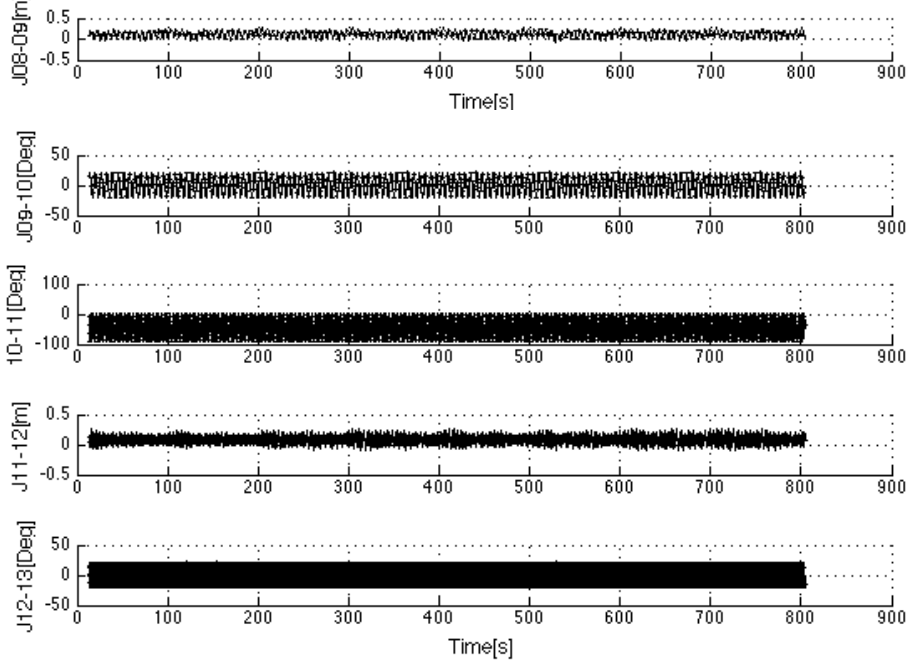

Fig. 6. Simulation results showing the sinusoidal position trajectories relative to each joint.

[2] A. Schiele and F. van der Helm, "Kinematic design to improve ergonomics in human machine interaction," Neural Systems and Rehabilitation Engineering, IEEE Transactions on, vol. 14, no. 4, pp. 456-469, Dec. 2006

[3] J. Kovecses, J. C. Piedboeuf, and C. Lange, "Dynamics modeling and simulation of constrained robotic systems," Mechatronics, IEEE/ASME Transactions on, vol. 8, no. 2, pp. 165-177, June 2003.

[4] M. Folgheraiter, M. Jordan, S. Straube, A. Seeland, S. Kim, and E. Kirchner, "Measuring the improvement of the interaction comfort of a wearable exoskeleton," International Journal of Social Robotics, vol. 4 no. 3, pp. 285-302, 2012.

[5] S. Feyzabadi, S. Straube, M. Folgheraiter, E. Kirchner, S. K. Kim, and
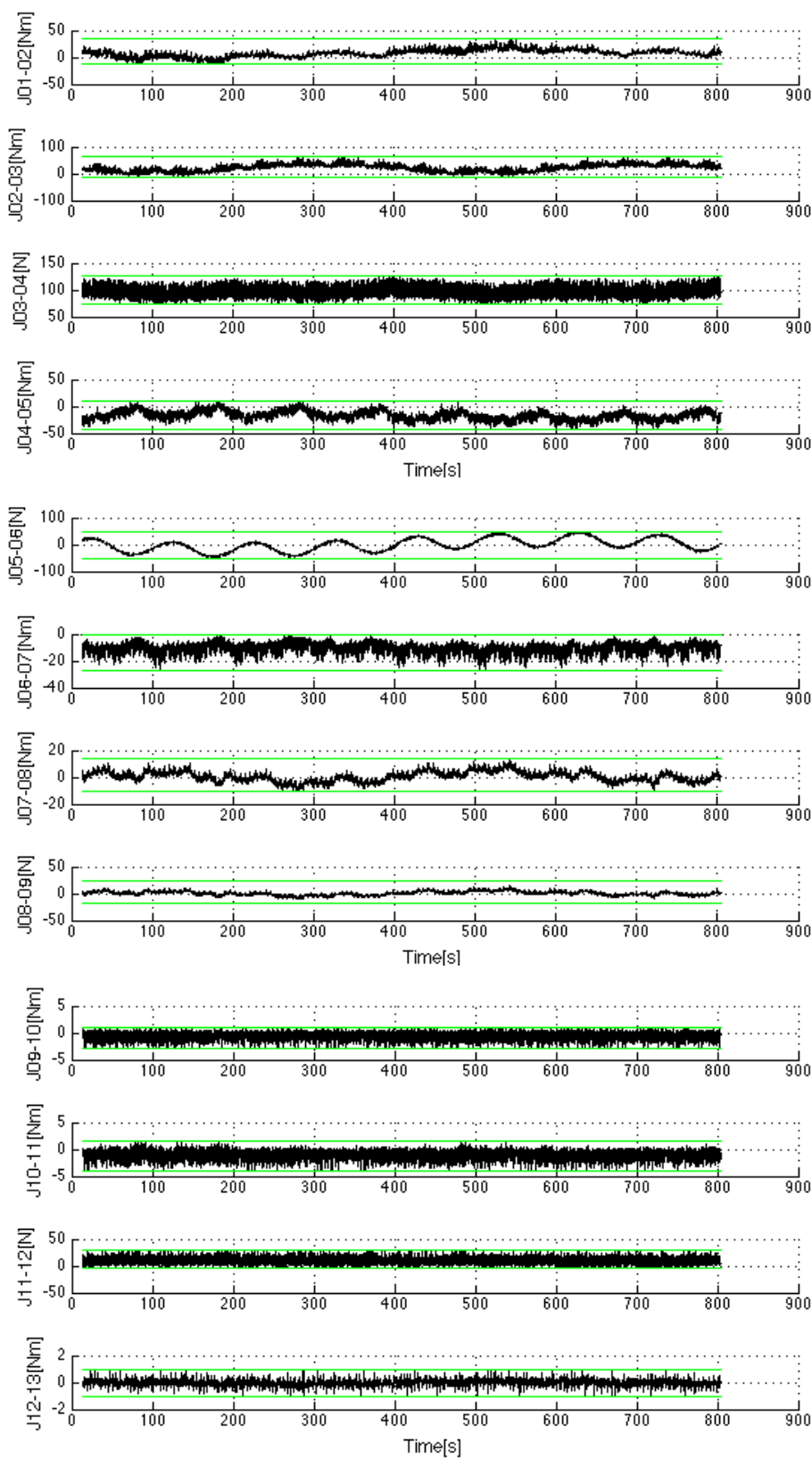

Fig. 7. Simulation results showing the resulting joints torques and forces with their limits in green.

J. Albiez, "Human force discrimination during active arm motion for force feedback design," Haptics, IEEE Transactions on, vol. 6, no. 3, pp. 309-319, July 2013.

[6] T. Huang, M. Li, Z. Li, D. Chetwynd, and D. Whitehouse, "Optimal kinematic design of 2-dof parallel manipulators with well-shaped workspace bounded by a specified conditioning index," Robotics and Automation, IEEE Transactions on, vol. 20, no. 3, pp. 538-543, June 2004.

[7] B. Bongardt, "Cad-2-sim - kinematic modeling of mechanisms based on the sheth-uicker convention," in 4th International Conference, (ICIRA11), Aachen, Springer, volume Part I, 2011, pp. 585-601. 